\title{
Exterior Companions to Hot Jupiters Orbiting Cool Stars Are Coplanar
}

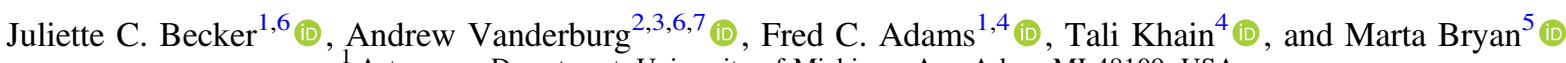 \\ Astronomy Department, University of Michigan, Ann Arbor, MI 48109, USA \\ ${ }^{2}$ Department of Astronomy, The University of Texas at Austin, 2515 Speedway, Stop C1400, Austin, TX 78712, USA \\ ${ }^{3}$ Harvard-Smithsonian Center for Astrophysics, 60 Garden Street, Cambridge, MA 02138, USA \\ ${ }^{4}$ Physics Department, University of Michigan, Ann Arbor, MI 48109, USA \\ ${ }^{5}$ California Institute of Technology, 1200 E California Boulevard, Pasadena, CA 91126, USA \\ Received 2017 August 19; revised 2017 October 2; accepted 2017 October 3; published 2017 November 16
}

\begin{abstract}
The existence of hot Jupiters has challenged theories of planetary formation since the first extrasolar planets were detected. Giant planets are generally believed to form far from their host stars, where volatile materials like water exist in their solid phase, making it easier for giant planet cores to accumulate. Several mechanisms have been proposed to explain how giant planets can migrate inward from their birth sites to short-period orbits. One such mechanism, called Kozai-Lidov migration, requires the presence of distant companions in orbits inclined by more than $\sim 40^{\circ}$ with respect to the plane of the hot Jupiter's orbit. The high occurrence rate of wide companions in hotJupiter systems lends support to this theory for migration. However, the exact orbital inclinations of these detected planetary and stellar companions is not known, so it is not clear whether the mutual inclination of these companions is large enough for the Kozai-Lidov process to operate. This paper shows that in systems orbiting cool stars with convective outer layers, the orbits of most wide planetary companions to hot Jupiters must be well aligned with the orbits of the hot Jupiters and the spins of the host stars. For a variety of possible distributions for the inclination of the companion, the width of the distribution must be less than $\sim 20^{\circ}$ to recreate the observations with good fidelity. As a result, the companion orbits are likely well aligned with those of the hot Jupiters, and the Kozai-Lidov mechanism does not enforce migration in these systems.
\end{abstract}

Key words: planets and satellites: dynamical evolution and stability - planets and satellites: gaseous planets

\section{Introduction}

Hot Jupiters, or Jupiter-sized planets orbiting with periods of a few days and distances of about $2 \%-5 \%$ of an astronomical unit, are an intriguing class of exoplanets with no analog in our own solar system. Although hot Jupiters are thought to account for only about $0.9 \%-1.5 \%$ of the total population of planets (Marcy et al. 2005; Cumming et al. 2008; Mayor et al. 2011; Wright et al. 2012; Wang et al. 2015), they are overrepresented in our current population of discovered exoplanets due to their large masses, large radii, and short orbital periods, which make them easy to detect in both transit and radial velocity observations. More than 300 hot Jupiters have been discovered to date. ${ }^{8}$

Since the discovery of the first hot Jupiters, understanding their origins has been a challenge for planet formation theorists, who have proposed several different mechanisms for how these planets form and how the systems are assembled into the architectures we see today. One traditional model for giant planet formation, which has been highly successfully applied to the formation of giant planets in our own solar system, is called core accretion (Stevenson 1982). In this model, a small core (likely composed of rocky and dense volatile materials) first forms in the protoplanetary disk, far enough away from its host star that dense volatile materials like water and/or methane are in solid, rather than gaseous, forms. Once a core has formed and become massive enough, it subsequently accretes a massive hydrogen/helium dominated envelope via runaway

\footnotetext{
${ }^{6}$ NSF Graduate Research Fellow.

7 NASA Sagan Fellow.

8 As of 2017 August 12, the NASA Exoplanet Archive has reported 315 known hot Jupiters. https://exoplanetarchive.ipac.caltech.edu/cgi-bin/TblView/ nph-tblView?app $=$ ExoTbls\&config $=$ planets.
}

gas accretion, leaving planets roughly the size and mass of Jupiter in orbits similar to that of Jupiter-far away from their host stars.

In this traditional picture, in order for the newly formed giant planet to become a hot Jupiter, it must then migrate inwards toward its host star, halting its migration at an orbital distance of about $0.05 \mathrm{au}$. Theorists have identified several mechanisms by which hot Jupiters might migrate from an orbit at tens of astronomical units into their present-day short-period orbits. One migration mechanism involves torques arising from tidaldisk interactions ("disk toques"), which could cause the hot Jupiters to slowly spiral inwards toward their host stars in the plane of the protoplanetary disk (see Tanaka et al. 2002; Kley $\&$ Nelson 2012). Another mechanism relies upon dynamical interactions between planets to excite high eccentricities in the proto-hot Jupiters after the gas disk has dissipated, bringing the planets into orbits whose perihelia distances are close to the surface of the host star. Tidal interactions when the planet comes close to the host star then might dissipate orbital energy, causing the orbit to shrink and result in the short-period orbits seen in hot-Jupiter systems. There are a couple of ways to excite these high eccentricities. Eccentricity can be excited via the Kozai-Lidov effect, which we call Kozai-Lidov migration (and which requires an inclined exterior companion; Lidov 1962; Kozai 1962). In some, more rare, cases, eccentricity can also be excited via low-inclination secular interactions, which we call coplanar high-eccentricity migration (Petrovich 2015).

Another recently revived mechanism for hot-Jupiter formation is in situ formation: instead of runaway accretion occurring far away from the host star, where dense volatile materials are abundant in their solid forms, the super-Earth-sized cores of the hot Jupiters form past the ice line, and migrate into their 
modern orbital radii simultaneously with other material in the disk. At this new orbital radius, the gas surface density would then be high enough for the core to experience runaway gas accretion at that location, forming a hot Jupiter (Batygin et al. 2016). This scenario builds on the idea that there exists a nearly ubiquitous population of super-Earth-sized planets orbiting close to their host stars (e.g., Fressin et al. 2013), many of which have sufficient mass to undergo runaway accretion.

These four distinct mechanisms for hot-Jupiter-system assembly (disk torques, coplanar high-eccentricity migration, Kozai-Lidov high-eccentricity migration, and in situ formation) have different observational outcomes. High-eccentricity migration destabilizes the orbits of close-in companions and requires the presence of distant massive companions in hotJupiter systems, which originally helped excite those high eccentricities. If Kozai-Lidov migration is dominant, then these companions should have mutual inclinations with the hot Jupiters of $\gtrsim 40^{\circ}$. By contrast, disk migration will likely result in dynamically quiet systems with low mutual inclinations. In situ formation initially produces a coplanar inner system, but subsequent secular interactions may eventually produce systems with either aligned (Batygin et al. 2016) or misaligned (Batygin et al. 2016; Spalding \& Batygin 2017) close-in exterior companions, such as those seen in the WASP-47 system (Becker et al. 2015). Such interactions would not change the natal stellar obliquity.

A powerful way to understand the architectures and formation histories of hot-Jupiter systems is through measurements of or constraints on the angles between the orbital angular momentum and the stellar spin axis. The difference between these angles is commonly called the stellar obliquity. There is a striking observed correlation between the photospheric temperature of the host star and the stellar obliquity. Observations of hot Jupiters (Winn et al. 2010a; Albrecht et al. 2012; and more tentatively, smaller planets as well, Mazeh et al. 2015) have shown that the orbits of planetary systems around cool stars $\left(T_{*}<6200 \mathrm{~K}\right)$ tend to be aligned with the spin of the host star, while the orbits of planets around hot stars $\left(T_{*}>6200 \mathrm{~K}\right)$ tend to be misaligned with the stellar spin axis. The boundary between the populations of hot and cool stars is commonly taken at stellar mass $M_{*}=1.3 M_{\odot}$, or equivalently at surface temperature $T_{*}=6200 \mathrm{~K}$. This threshold is often called the "Kraft break" (Kraft 1967; van Saders \& Pinsonneault 2013). This mass limit corresponds to stellar configurations where the convective envelope becomes thin, which provides some clues to the physical processes involved.

Although the observed pattern of obliquities as a function of stellar surface temperature remains under study as additional stellar obliquity measurements are performed with methods such as Doppler tomography (recent measurements include Johnson et al. 2017; Zhou et al. 2017) or the RossiterMcLaughlin technique (Ohta et al. 2005), the fact that hot Jupiters around cool stars tend to have orbits that are well aligned with their host stars' spins axes appears to hold. However this alignment came about, it is difficult to produce it by random chance, and similarly difficult to reproduce it once it has been disturbed. This alignment could be primordial (for example, magnetic fields can realign a young star with its disk; see Spalding \& Batygin 2015), or it could come about by realignment of stellar spin axes due to the planets' tidal influence (Hut 1980; Albrecht et al. 2012; Adams \& Bloch
2015), a fairly slow process, which takes hundreds of millions of years or more (Albrecht et al. 2012; Lai 2012). Therefore, in order for hot Jupiters to maintain their spin/orbit alignment, their obliquities cannot be perturbed or changed on timescales significantly shorter than this benchmark value.

In this paper, we ask the question, "What effect do distant perturbing bodies have on the alignment of hot-Jupiter orbits and the spins of their host stars?" Many distant companions, both planetary and stellar, to hot Jupiters have been found, and in fact are more frequent around hot-Jupiter hosts than around typical stars (Knutson et al. 2014; Ngo et al. 2015; Bryan et al. 2016). These companions also seem to have little effect on the hot Jupiters' spin-orbit alignments (Knutson et al. 2014; Ngo et al. 2015; Bryan et al. 2016). But if these distant companions have a strong enough gravitational influence on the hot Jupiters and have large mutual inclinations, they could in principle disturb the spin-orbit alignment of the hot Jupiters away from what we observe in cool stars. By calculating the effect of the observed distant companions to hot Jupiters, we can place constraints on the mutual inclination between these companions and the well aligned hot Jupiters.

Here, we statistically constrain the orbital inclinations of exterior long-period companions in hot-Jupiter systems. We approach this problem by identifying a sample of hot Jupiters orbiting cool stars with known long-period companions and measured stellar obliquity and calculating the probability that each of these hot Jupiters will retain its low inclination as a function of the inclination of the distant perturbing companions using secular and $N$-body techniques. In Section 2, we describe our sample selection and analysis techniques. In Section 3, we present the statistical results of our analysis and show that most companions of hot Jupiters around cool stars orbit near the plane of the hot Jupiters' orbits. In Section 4, we discuss the implications of this result on hot-Jupiter formation and suggest avenues for future work.

\section{Methods}

\subsection{Sample Selection}

We focus in this paper on transiting hot Jupiters with known companions detected via radial velocity observations. Since the hot Jupiters transit, it is often possible to measure components of the stellar obliquity via the Rossiter-McLaughlin effect, a crucial ingredient in our calculations. Also, because the hot Jupiters transit, we know their orbital inclinations quite precisely to be nearly $90^{\circ}$. Therefore, any constraint on the orbital inclination of the distant companion constrains the mutual orientation of the two planets' orbits.

The systems we consider in this work are those with the following properties.

1. The host star is cool (with an effective temperature below Kraft break; $\left.T_{*}<6200 \mathrm{~K}\right)$.

2. The star hosts a hot-Jupiter (roughly Jupiter-mass planet with an orbital period between 0.8 and 6.3 days; as defined in Steffen et al. 2012).

3. There exists in the literature a measure of either the projected or true stellar obliquity (angle between the stellar spin axis and the planet's orbital angular momentum vector) for the host star. We do not require this obliquity to have any particular value or precision, but merely for a measurement to exist. 


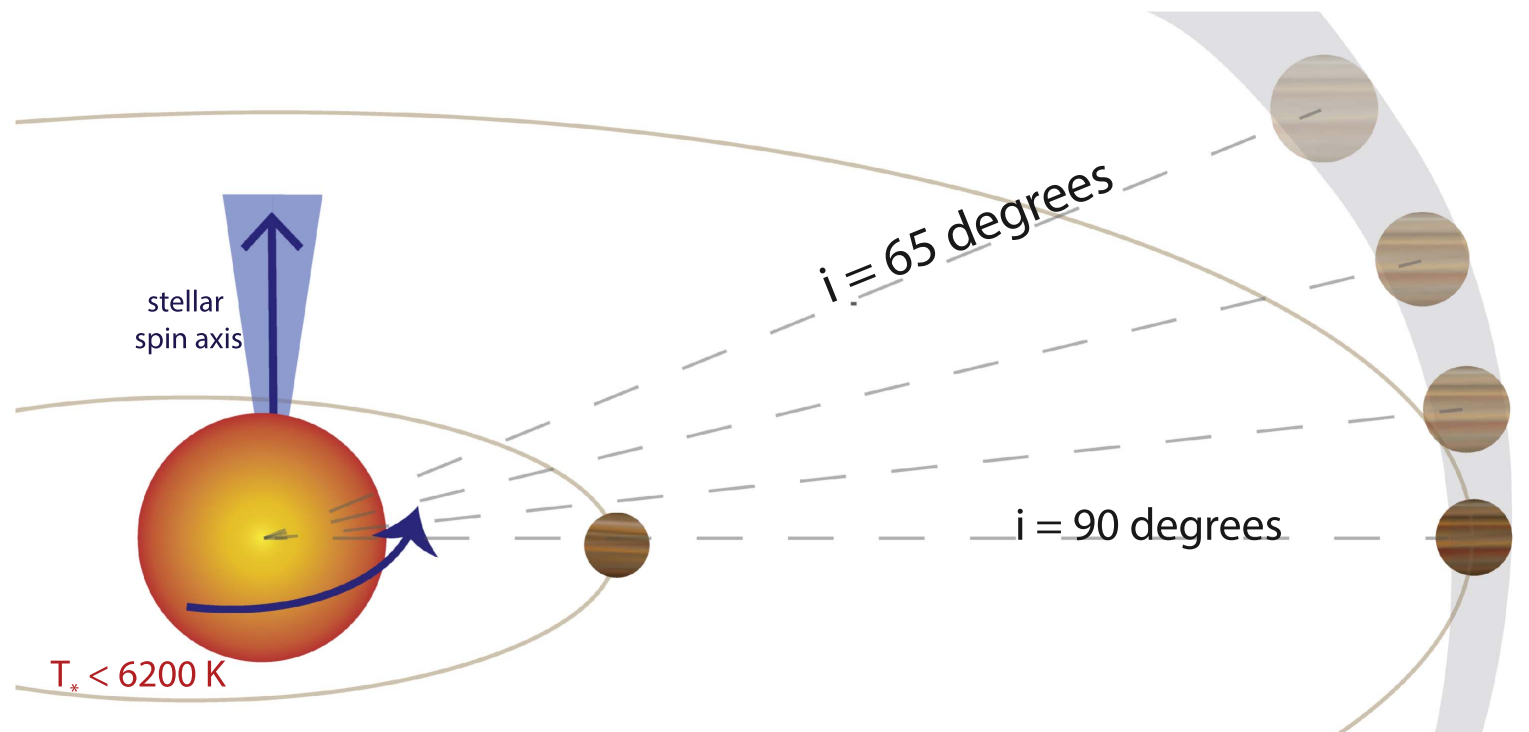

Figure 1. Schematic diagram showing the orbital architecture of systems considered in this work. The systems we consider are those with stellar effective temperatures below the Kraft break $\left(T_{*}<6200 \mathrm{~K}\right)$, a measured stellar obliquity, and evidence of an exterior companion whose residuals do not correlate with stellar activity level. The inclination of the outer companion is not known for any of the systems in our sample (this quantity is varied in the analysis).

Table 1

Orbital Parameters Used for the Analysis in This Work, with the Literature Sources for Each Measured Value

\begin{tabular}{|c|c|c|c|c|c|c|}
\hline & HAT-P-4 & HAT-P-13 & WASP-22 & WASP-41 & WASP-47 & WASP-53 \\
\hline \multicolumn{7}{|c|}{ Stellar Properties } \\
\hline$M_{*}\left(M_{\odot}\right)$ & $1.26 \pm 0.10$ & $1.22_{-0.10}^{+0.05}(5)$ & $1.249_{-0.17}^{+0.088}(7)$ & $0.987 \pm 0.047(7)$ & $1.00 \pm 0.05(13)$ & $0.87 \pm 0.08$ \\
\hline$R_{*}\left(R_{\odot}\right)$ & $1.617_{-0.05}^{+0.057}$ & $1.281 \pm 0.079$ & $1.255_{-0.034}^{+0.035}(7)$ & $0.886 \pm 0.017(7)$ & $1.15 \pm 0.04$ & $0.96 \pm 0.24(17)$ \\
\hline$T_{*}(\mathrm{~K})$ & $5860 \pm 80$ & $5653 \pm 90(5)$ & $6153 \pm 50(7)$ & $5546 \pm 33(7)$ & $5400 \pm 100(16)$ & $4950 \pm 60(17)$ \\
\hline \multicolumn{7}{|c|}{ Hot-Jupiter Properties } \\
\hline$e_{b}$ & $0(1,2)$ & $0.0133 \pm 0.0041$ & $0.023 \pm 0.012$ & $<0.026(11)$ & $0.0028 \pm 0.0028$ & $<0.03$ (17) \\
\hline$i_{b}(\mathrm{deg})$ & $88.76_{-1.38}^{+0.89}$ & $83.4 \pm 0.6(5)$ & $89.2 \pm 0.5(8)$ & $89.4_{-0.3}^{+0.3}(11)$ & $89.2_{0.7}^{0.5}$ & $87.08_{-0.15}^{+0.16}(17)$ \\
\hline$\omega_{b}(\operatorname{deg})$ & $\cdots$ & $181 \pm 45$ & $27_{-78}^{51}$ & $\cdots$ & $51 \pm 82$ & $\cdots$ \\
\hline \multicolumn{7}{|c|}{ Companion Properties } \\
\hline$e_{c}$ & $(3,4)$ & $0.6616 \pm 0.0054(6)$ & $(3,4)$ & $0.294 \pm 0.024$ & $0.13 \pm 0.10$ & (17) \\
\hline$\omega_{c}(\operatorname{deg})$ & $(3,4)$ & $176.7 \pm 0.5$ & $(3,4)$ & $353 \pm 6(11)$ & $144 \pm 53(11)$ & (17) \\
\hline
\end{tabular}

${ }^{a}$ This companion does not have a fitted orbit, but a trend indicating the presence of a companion with an orbital period longer than our observational baseline. In this work, we sample the orbital elements of these companions from the posteriors provided in Bryan et al. (2016).

${ }^{\mathrm{b}}$ Using the methodology in Knutson et al. (2014) and Bryan et al. (2016), we create a posterior of the same style for WASP-53, using the radial velocity measurements from Triaud et al. (2017).

References. (1) Kovács et al. (2007); (2) Winn et al. (2011); (3) Knutson et al. (2014); (4) Piskorz et al. (2015); (5) Bakos et al. (2009); (6) Winn et al. (2010b); (7) Southworth et al. (2016); (8) Anderson et al. (2011); (9) Maxted et al. (2010); (10) Maxted et al. (2011); (11) Neveu-VanMalle et al. (2016); (12) Vanderburg et al. (2017); (13) Weiss et al. (2017); (14) Becker et al. (2015); (15) Sanchis-Ojeda et al. (2015a); (16) Hellier et al. (2012); (17) Triaud et al. (2017).

4. There is evidence in the literature that the system has an additional companion in the system; this companion may be a Jupiter-like planet or a brown dwarf.

Figure 1 illustrates the geometry of the type of systems we consider in this work. A list of all the stars that fit these criteria and their properties, as well as the measured orbital properties of their planets, is given in Table 1. Additional companions in these systems come in two forms. First, there are systems for which the orbits of additional companions have been wellcharacterized, and the period of their orbits are known (such as WASP-41, WASP-47, and HAT-P-13). Second, there are systems in which a trend in the RV data has been identified, but 
the (putative) companion does not have a precisely measured period (such as HAT-P-4, WASP-22, and WASP-53). These latter systems only have constraints on the companion's orbits (see, for example, Figure 10 of Knutson et al. 2014), which can be derived from the radial velocity curves. In this work, we use the posteriors from Bryan et al. (2016) for HAT-P-4 and WASP-22, and generate a new posterior for WASP-53 using the data in Triaud et al. (2017) and the method from Bryan et al. (2016), without any adaptive optics constraints on outer companions.

We exclude from our sample stars with companions and effective temperatures measured to be above the Kraft break. HAT-P-7, HAT-P-32, and HAT-P-2 have temperatures right above Kraft break and have high projected obliquities, which is consistent with the convective realignment argument (the stars did not have a sufficient convective envelope to become realigned early in their lives). We exclude warm Jupiters (defined using the definition given in Steffen et al. 2012 to be Jupiter-mass planets with orbital periods between 6.3 days and 15.8 days) because these objects are not typically proposed to form through a high-eccentricity pathway and therefore, unlike hot Jupiters, are not expected to possess inclined companions (Huang et al. 2016).

The system XO-2N contains a hot Jupiter (Burke et al. 2007), orbits a cool star, and has a projected stellar obliquity of $7^{\circ} \pm 11^{\circ}$ (Damasso et al. 2015). Knutson et al. (2014) also presented RV evidence of a long-period signal in the system. However, Damasso et al. (2015) found a correlation between the RV residuals and the stellar activity index $R_{\mathrm{HK}}$, indicating that the long-period signal is likely stellar activity and not a companion. For this reason, we also exclude this system from our sample (although we note that this system and its companion would fit perfectly into the aligned paradigm we see in our sample, were the companion to be real).

Of the systems we include, some have additional components that do not significantly effect the evolution of the system. The WASP-47 system is unique among hot-Jupiterhosting systems because it contains two short-period planets in addition to the hot Jupiter WASP-47b. Both of these additional planets are roughly coplanar with the hot-Jupiter orbit (as they were both discovered via $\mathrm{K} 2$ transit photometry). In this work, we consider only the precession of the hot Jupiter, and do not impose additional constraints based on the transiting behavior or potential dynamical instability of the other planets (unlike the analyses done in Becker \& Adams 2017; Vanderburg et al. 2017). We choose to consider the hot Jupiter alone because it is the planet for which the Rossiter-McLaughlin measurement was made (Sanchis-Ojeda et al. 2015b). Excluding WASP-47 from the sample due to its unusual architecture would not change the results significantly since all hot Jupiters in our sample are aligned, so to maximize our sample size, we choose to include it.

HAT-P-13 actually has three planets, a hot Jupiter and its two companions. The first companion has a period of $428.5 \pm 3.0$ days, an eccentricity of $0.691 \pm 0.018$, and an $m \sin i$ of $15.2 \pm 0.1 M_{J}$ (Bakos et al. 2009). The second companion does not have a measured period, but an RV trend indicates its existence (Winn et al. 2010b). The inner of those two (the middle body in the system) does not transit. Since the perturbations from the outermost body are expected to be adiabatic (Becker \& Batygin 2013), we ignore the effect of the outer planet in our analysis. We do note that the influence of the outer planet has the potential to adiabatically misalign both inner planets. However, given that we measure a low stellar obliquity, and will show later that the middle planet is probably also aligned, it is unlikely the outer companion is highly inclined. Future modeling efforts may readily test this prediction.

\subsection{The Laplace-Lagrange Secular Model}

Additional exterior companions can alter the orbital inclination of the inner hot Jupiter through planet-companion interactions. As these interactions are mainly secular and nonresonant, we can approximate the system's orbital evolution over time using secular Laplace-Lagrange theory. This provides an approximation for the expected effect, which can be used to guide further analysis. Although we also use numerical $N$-body simulations (see below) to construct our final results in this work, this section outlines the analytic, guiding theory for describing the inclination evolution over time.

As we expect secular interactions to dominate, we can construct a disturbing function for the planetary system, excluding terms that depend on the relative positions of the planets in their orbits (Murray \& Dermott 1999). The result is an equation that treats the planets as smeared-out rings of mass. Including only the terms describing the inclination of each planet's orbit to second order, this result becomes

$$
\mathcal{R}_{j}^{(\mathrm{sec})}=n_{j} a_{j}^{2}\left[\frac{1}{2} B_{j j} I_{j}^{2}+\sum_{k=1, j \neq k}^{N}\left(B_{j k} I_{j} I_{k} \cos \left(\Omega_{j}-\Omega_{k}\right)\right)\right],
$$

where $j$ is the planet number, $n$ is the mean motion, $I$ is the inclination, $\omega$ is the argument of pericenter, and $\Omega$ is the longitude of the ascending node. In the case of a spherical central body, the quantities $B_{i j}$ represent the interaction coefficients between pairs of planets and are given by

$$
B_{j j}=-n_{j}\left[\frac{1}{4} \sum_{k=1, j \neq k}^{N} \frac{m_{k}}{M_{c}+m_{j}} \alpha_{j k} \bar{\alpha}_{j k} b_{3 / 2}^{(1)}\left(\alpha_{j k}\right)\right],
$$

and

$$
B_{j k}=n_{j}\left[\frac{1}{4} \frac{m_{k}}{M_{c}+m_{j}} \alpha_{j k} \bar{\alpha}_{j k} b_{3 / 2}^{(1)}\left(\alpha_{j k}\right)\right],
$$

where $m_{k}$ is the mass of the $k$ th planet, $M_{c}$ is the mass of the central star, the $\alpha_{j k}$ are the semimajor axis ratios $a_{j} / a_{k}$, and $\bar{\alpha}_{j k}$ are the semimajor axis ratios for $a_{j} / a_{k}<1$. The function $b_{3 / 2}^{(1)}(\alpha)$ is the Laplace coefficient, which is defined by

$$
b_{3 / 2}^{(1)}(\alpha)=\frac{1}{\pi} \int_{0}^{2 \pi} \frac{\cos \psi d \psi}{\left(1-2 \alpha \cos \psi+\alpha^{2}\right)^{3 / 2}} .
$$

Further explanation of this theory and potential expansions of the model can be found in Murray \& Dermott (1999). Using the standard transformation

$$
p_{j}=I_{j} \sin \Omega_{j} \quad \text { and } \quad q_{j}=I_{j} \sin \Omega_{j},
$$

the solutions of the eigenvalue problem defined by matrix $\boldsymbol{B}$ take the form

$$
p_{j}(t)=\sum_{k=1}^{N} I_{j k} \sin \left(f_{k} t+\gamma_{k}\right)
$$


and

$$
q_{j}(t)=\sum_{k=1}^{N} I_{j k} \cos \left(f_{k} t+\gamma_{k}\right) .
$$

To complete the initial condition problem, we define normalized eigenvectors $\mathcal{I}_{j k}$ and corresponding scaling factors $T_{k}$ for the eigenvectors $I_{j k}$,

$$
I_{j k}=T_{k} \mathcal{I}_{j k},
$$

which allows us to use Equations (6) and (7) combined with the initial values of the inclination angles $I_{j}$ and the angles $\Omega_{j}$ for each planet to solve for the scaling factors $T_{k}$, i.e.,

$$
p_{j}(t=0)=\sum_{k=1}^{N} T_{k} \mathcal{I}_{j k} \sin \gamma_{k}
$$

and

$$
q_{j}(t=0)=\sum_{k=1}^{N} T_{k} \mathcal{I}_{j k} \cos \gamma_{k}
$$

The result is an expression defining the time evolution of the orbital inclination of each body in the system,

$$
I_{j}(t)=\sqrt{\left[p_{j}(t)\right]^{2}+\left[q_{j}(t)\right]^{2}} .
$$

This equation can be used to generate the inclination evolution for planets in a system dominated by secular effects. By inspection, we see that the total angular momentum direction in the system will be conserved, but traded between planets in amounts mediated by the magnitude of the interaction coefficients. An example of the application of this theory is shown in Figure 2, which plots the orbital inclination angles (as computed with the Laplace-Lagrange secular theory detailed above) over time for two realizations of WASP-41b and WASP-41c. The first system is considered to be coplanar, whereas the second case assumes that the companion WASP$41 \mathrm{c}$ is slightly inclined. We note that when Equation (11) is used, the initial inclinations of transiting planets should be set to $0^{\circ}$, rather than the $90^{\circ}$ traditionally reported observationally to denote edge-on orbits, due to the small angle approximation used in deriving the secular equations.

An inclined companion leads the orbit of hot Jupiter (-41b) to precess and allows the inclination angles to oscillate over time. A precessing hot Jupiter will appear aligned with its host's spin axis some (small) fraction of the time. This exact value depends on the observational error on the obliquity measurement as well as the orbital elements of all bodies in the system. As a result, for a single system, the fact that a hot Jupiter is aligned with the stellar spin axis does not completely specify the inclination of the companion. It is possible that our observations happen to occur at a moment in the secular cycles where the system passes through alignment. However, if we observe the entire population of hot-Jupiter hosts to have spin axes aligned with their hot Jupiter's orbital angular momentum, then it is unlikely that their companions are highly inclined. In other words, the assessment of alignment in hot-Jupiter systems must be done statistically.

\subsection{Numerical Computation}

In addition to the secular theory described above, we ran numerical $N$-body integrations of these systems, as such simulations are capable of recovering orbital behavior that is
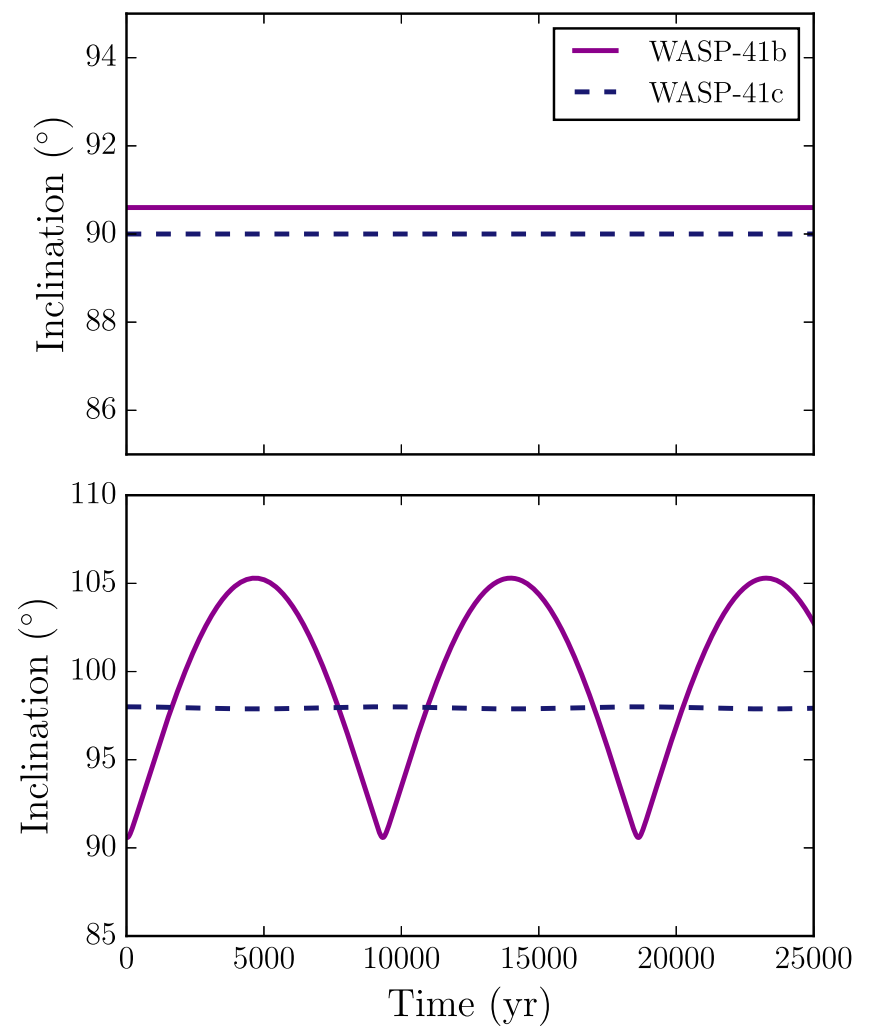

Figure 2. Evolution of the inclination of WASP-41b and WASP-41c as given by Laplace-Lagrange secular theory: the secular theory was computed using inclinations centered around $0^{\circ}$, and the inclination plotted is the secular result plus $90^{\circ}$ (to signify that WASP-41b is seen in an edge-on orbit). The companion's observed initial inclination differs in the two panels: $i_{c}=90^{\circ}$ (top panel) and $i_{c}=98^{\circ}$ (bottom panel). The presence of an inclined companion (-41c) results in an oscillating inclination angle for the hot Jupiter (-41b), affecting its angular momentum direction. In particular, a higher inclination of WASP-41c decreases the amount of time WASP-41b spends near its original orbital momentum direction, thereby increasing the likelihood of observing obliquity misalignments.

not apparent from the secular theory alone. In Figure 3, we show the comparison between the results computed using each method for one system in our sample (WASP-41, the same system visualized in Figure 2). The most important differences between the secular and numerical approaches are as follows. (1) The secular approach does not detect dynamical instabilities that result in ejections or collisions (see Figure 3-the points that lie on the $x$-axis are points where such a dynamical instability occurred, the inner planet was lost, and thus the system would never recreate observations). (2) The numerical approach allows for time-dependence in the semimajor axis, while the secular theory does not. (3) The numerical approach will correctly capture the behavior of mean motion resonances should they arise (although, we expect these to be rare for the particular geometry of this problem). The differences between the secular and numerical results in Figure 3 demonstrate that the secular theory is a good but not perfect approximation. To encompass all of these behaviors, we treat the secular theory as motivation, and examine the behavior of each of the six systems in our sample using $N$-body integrations. In this numerical work, we use the system parameters and posteriors presented in Table 1.

Another reason to use $N$-body methods rather than the secular approximation is that, although the numerical computations are very expensive, we only have six systems in our 


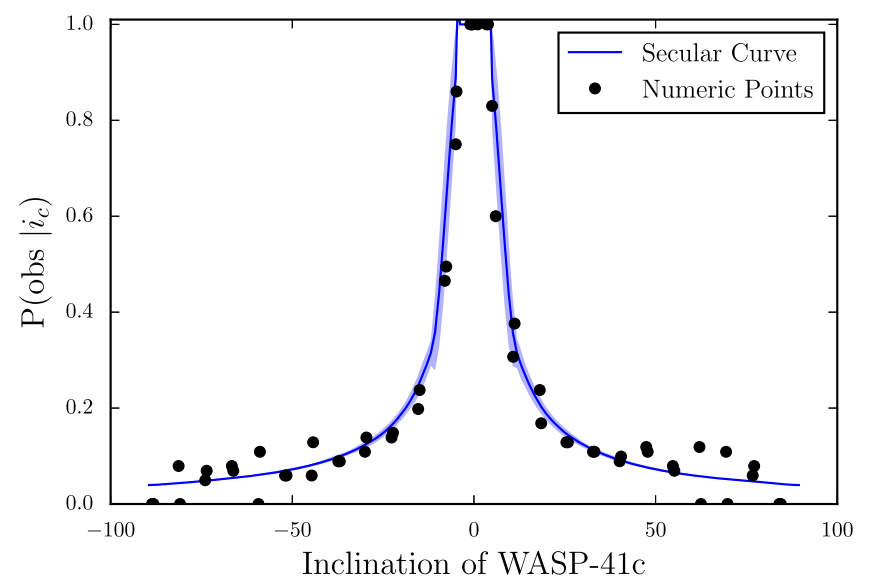

Figure 3. Distribution for the probability of recreating the observations (obs), given some companion inclination for WASP-41c $\left(i_{c}\right)$. This plot shows the comparison between the results computed from secular theory (solid blue curve) and the numerical $N$-body results (points). Here, an inclination of $0^{\circ}$ denotes an edge-on orbit (which observers report as having $i=90^{\circ}$ ). Except for the method used to generate the time series of orbital elements (secular theory vs. the Mercury6 $\mathrm{N}$-body integrator), the probabilities in each case were generated the same way. The numerical simulations show good agreement with the secular calculation. The secular theory provides a robust motivation for this problem, and can be used to predict the interactions between planets at low mutual inclinations.

sample, and thus the calculation is feasible. Notice that, on average, a single trial using a Mercury $6 \mathrm{~N}$-body simulation takes $10^{3}-10^{4}$ longer than the corresponding pythongenerated secular evolution. For longer integration times, this discrepancy grows larger. Future analyses with a larger sample size might be able to use the secular approximation, which is generally accurate for sufficiently small mutual inclinations.

The purpose of these numerical experiments is to examine the effect that varying the inclination of the companion has on the alignment of the spin axis of the star and the orbital angular momentum vector direction of the hot Jupiter. Recall that for a single system, we cannot draw firm conclusions about its orbital geometry from the fact the hot Jupiter transits today because precessing orbits allow planets to transit from a given line of sight with some duty cycle. Similarly, we must make an assumption about the underlying companion inclination distribution. Since we are testing the population as a whole, and not just individual systems, we consider three possible priors for the population of companion inclinations: a Fisher distribution, a uniform distribution, and a delta function. For each distribution, we assign the width of the distribution to be $\sigma$, when $\sigma^{2}=\left\langle\sin ^{2} i\right\rangle$, and the functional forms of each probability distribution $d p=f d i$ and width are given as follows.

1. Fisher distribution. The Fisher distribution is often used (Fabrycky \& Winn 2009; Tremaine \& Dong 2012) to describe the inclinations of planetary orbits, especially relative to the spin axis of their host star (Morton \& Winn 2014, see this paper for some illustrative plots describing the Fisher distribution). Its functional form can be written as

$$
f_{f}(i \mid \kappa)=\frac{\kappa}{2 \sinh \kappa} e^{\kappa \cos i} \sin i
$$

when $i$ is the orbital inclination angle. Then, we can find the width $\sigma$ :

$$
\begin{aligned}
\sigma^{2} & =\left\langle\sin ^{2} i\right\rangle=\int f_{\theta}(\theta \mid \kappa) \sin ^{2} i d i \\
& =2 \frac{\operatorname{coth} \kappa}{\kappa}-\frac{2}{\kappa^{2}}
\end{aligned}
$$

or

$$
\sigma=\sqrt{2 \frac{\operatorname{coth} \kappa}{\kappa}-\frac{2}{\kappa^{2}}} .
$$

This form reduces to a Rayleigh distribution for large $\kappa$. For $\kappa \rightarrow 0$, the distribution becomes approximately isotropic.

2. Uniform distribution. We assume that all companions come from a population with uniform scatter, but some maximum allowed inclination (defined as $\theta_{m}$ ). For each iteration, we generate companions by drawing from a uniform inclination distribution between a $0^{\circ}$ mutual inclination and some maximum inclination. The functional form for this distribution can be written as

$$
\frac{d p}{d i}=f_{u}\left(i \mid \theta_{m}\right)=\frac{1}{2 \theta_{m}} .
$$

The width $\sigma$ of this distribution is again defined by the expectation value of $\sin ^{2} i$, where $i$ is the inclination drawn for each trial:

$$
\sigma^{2}=\left\langle\sin ^{2} i\right\rangle=\int f_{u} \sin ^{2} i d i .
$$

For a distribution ranging between $-\theta_{m}$ and $\theta_{m}$ :

$$
\sigma^{2}=\int_{-\theta_{m}}^{\theta_{m}} \frac{1}{2 \theta_{m}} \sin ^{2} i d i=\frac{\theta_{m}-\cos \theta_{m} \sin \theta_{m}}{2 \theta_{m}},
$$

or

$$
\sigma=\sqrt{\frac{1}{2}-\frac{\sin 2 \theta_{m}}{4 \theta_{m}}} .
$$

3. Delta function distribution. We assume that all companions have the same inclination-so, the underlying companion distribution is a delta function at some inclination. This distribution has the probability function

$$
\frac{d p}{d i}=f_{\delta}\left(i \mid \theta_{x}\right)=\delta\left(i-\theta_{x}\right)
$$

and the width $\sigma$ can also be found:

$$
\sigma^{2}=\left\langle\sin ^{2} i\right\rangle=\int f_{\delta} \sin ^{2} i d i=\sin ^{2} \theta_{x} .
$$

So, the final width to the delta function companion distribution will be

$$
\sigma=\sin \theta_{x}
$$

For each of those three priors, we initialized 1000 connected realizations of each of the six systems. (For example, a single realization includes all six planetary systems in independent integrations, all of which have inclinations drawn from the Fisher distribution of a given width. This process is then repeated 1000 times with different distribution widths. Then, the entire set of 1000 is repeated for each other prior type). In each realization, we sample from the known posteriors for each 

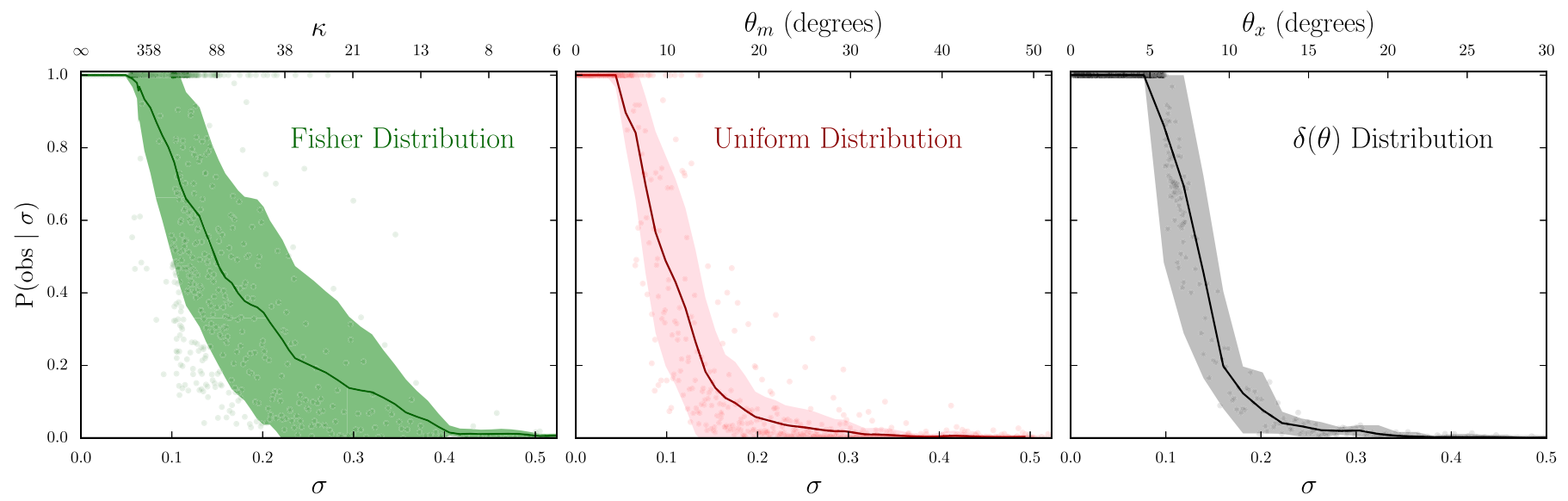

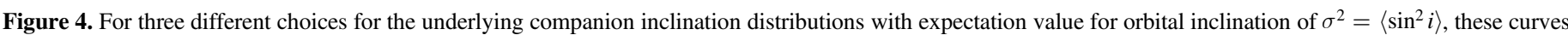

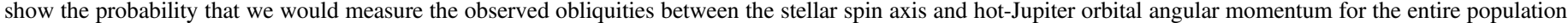

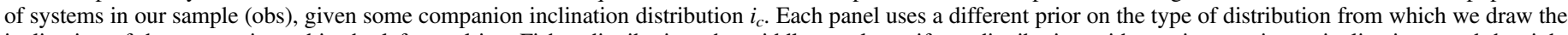

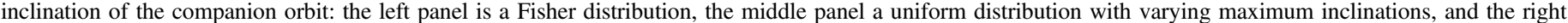

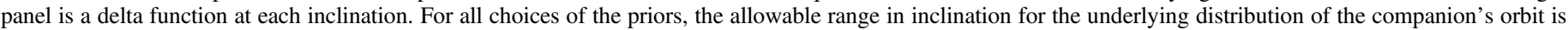
less than $\sim 20^{\circ}$ out of the plane containing the hot Jupiter.

orbital element for all known planets. For the orbital elements of the hot Jupiter in each system, we set its initial inclination to be $90^{\circ}$, and draw its orbital period, mass, eccentricity, and argument of periastron from observational priors (see Table 1). For the orbital elements of the perturbing companions, we assign their orbital periods, masses, eccentricities, and arguments of periastron in the same way. We also draw an inclination for the perturbing companion from the prior being tested (either Equations (12), (15), or (19)). If a planet has an $m \sin i$ measurement instead of a true mass, we combine this measurement with our drawn inclination to find the true mass of the companion for that realization.

After the initial conditions for the systems are specified, we evolve the systems using Mercury6 (Chambers 1999) with time-steps set initially to be $1 \%$ the orbital period of the innermost planet, and use the hybrid symplectic and Bulirsch-Stoer (B-S) integrator. We require energy conservation to a part in $10^{-8}$ or better, and allow each integration to run for $10 \mathrm{Myr}$ (which encompasses many secular periods) and include the effects of GR.

For each set of six systems, we then use the time series of orbital elements computed by the $\mathrm{N}$-body simulations to compute the projected stellar obliquity at each time-step. As each star in our sample has an observationally measured projected obliquity, we then compute the probability that our simulated stellar obliquity would be measured to be consistent with this value (assuming the observational errors on our simulated measurement are equal to the error on the true measurement; see the third row of Table 1 for the projected obliquities and errors). The result of this computation is the probability that an observer would observe the stellar obliquity to be consistent with the true value we measure observationally at the current epoch.

Since each realization we have simulated includes six integrations (one for each planetary system), we then compute for each time-step the product of these six individual probabilities. This product is the probability that a simulated telescope making an observation at that time-step would measure a set of six projected stellar obliquities consistent with the true, current-day values. Then, using the entire time series of probabilities, we compute a single marginalized probability $P(\operatorname{obs} \mid \sigma)$, when $\sigma$ is computed directly from the functional form of each prior (the final forms of which are given in
Equations (14), (13), and (21)). This single probability describes the chance that we would observe all the same stellar obliquities presented in the third row of Table 1 given the prior we chose for the underlying distribution of companion inclinations. We also plot in Figure 4 a smoothed curve representing the mean probability for each distribution width, with contoured error bars representing the $1 \sigma$ scatter at each distribution width.

\section{Results}

\subsection{The Companion Population Tends to have Nearly Coplanar Orbits}

Figure 4 illustrates the main result of this work. We have considered systems containing hot Jupiters orbiting cool stars for which an obliquity measurement exists and which exhibit evidence for a companion. For three different types of distributions for the (unknown) inclination angle of the companion orbit, the numerical $N$-body simulations show that a large fraction of the cases with large mutual inclination angles result in a low probability of recreating the observations. As a result, it is unlikely that the companions to these cool hotJupiter hosts generally have a high mutual inclination. Indeed, for all three prior choices (which range from the restrictive delta function distribution to the physically motivated and commonly used Fisher distribution), the allowable range of orbital planes for the companions is within $20^{\circ}-30^{\circ}$ of the orbital plane of the hot Jupiter: the probability curves in Figure 4 are plotted against $\sigma=\sqrt{\left\langle\sin ^{2} i\right\rangle}$, and the top axis of each panel presents for physically intuitive units for each prior (the definitions of which can be found in Section 2.3). From these curves, we can compute the $95 \%$ confidence interval for each prior, which will define an upper limit on the value we can expect $\sqrt{\left\langle\sin ^{2} i\right\rangle}$ to assume, and then convert this to an angle, $i_{c}$, describing the likely maximum misalignment of exterior, coupled companions in these systems. For the Fisher distribution, this value is $i_{c} \sim 24^{\circ}$. For the uniform distribution, this value is $i_{c} \sim 13^{\circ}$. For the delta function distribution, this value is $i_{c} \sim 13^{\circ}$.

Although the sample of known hot Jupiters with both stellar obliquity measurements and known exterior companions is 
small (only six such systems have been discovered at the time that this paper was written), we can nonetheless make significant inferences on the underlying distribution of possible orbital inclinations for the population of companions. The dynamical calculations carried out here show that, through primarily secular evolution, the inclination angles of the orbits are expected to evolve in the presence of an inclined companion. The fact that the stellar obliquity with respect to the hot Jupiter tends to be low constrains the secular evolution histories in these systems. If the underlying population of companions to systems containing hot Jupiters around cool stars has a random distribution of uniformly distributed inclination angles, the chance that our observations happened to catch the six known systems at times where the orbits of the hot Jupiters are aligned with the stellar spin axis is only $\sim 10^{-7}$. As shown in Figure 4, the orbits of the underlying companion population in these hot-Jupiter systems are likely to be confined near the plane of the hot-Jupiter orbit.

\subsection{Implications for Hot-Jupiter Formation and Migration}

Our conclusion that orbits of distant exterior companions to hot Jupiters are likely coplanar with hot Jupiter orbits has important implications for migration scenarios. The narrow distribution of inclination angles inferred here favors diskdriven migration mechanisms for hot Jupiters around cool stars. In this case, the disk and planets remain in nearly the same plane, and the disk is generally aligned (within about $30^{\circ}$ ) with the stellar spin axis (for additional discussion, see Lee et al. 2014; Becker et al. 2015; Huang et al. 2016; Weiss et al. 2017 for discussions of alignment, and Lai et al. 2011; Lai 2014; Spalding et al. 2014; Fielding et al. 2015 for mechanisms to excite misalignments, particularly with systems around hot stars). In situ formation of hot Jupiters would also lead to well aligned planetary orbits (Batygin et al. 2016). In contrast, higheccentricity migration does not generally lead to low mutual inclinations. In this latter scenario, the migrating hot Jupiter attains high eccentricity, and hence a small periastron, so that tidal dissipation can circularize the orbit with a small semimajor axis. The mechanisms invoked to excite the high eccentricity-including the Kozai-Lidov effect from stellar companions, planet-planet scattering, and secular interactions between planets-generally result in high-inclination configurations (Fabrycky \& Tremaine 2007; Nagasawa et al. 2008; $\mathrm{Naoz}$ et al. 2011). As these high-inclination configurations are found in hot-Jupiter systems around hot stars $\left(T_{*}>6200 \mathrm{~K}\right)$, it is possible either that (a) hot stars, lacking a convective envelope, fail to realign the stellar spin-axis with orbital angular momentum early in their lives, or (b) the systems orbiting hot stars assemble via a different pathway. In either case, for cool stars, we favor a disk-driven migration scenario for dynamically coupled companions.

On the other hand, some exceptions are possible (Petrovich 2015), and the number of hot-Jupiter systems for which we can carry out the analysis of this paper remains small. Fortunately, future observations should find an increasing number of hotJupiter systems with additional companions orbiting cool stars. If these upcoming observations find a large number of misaligned systems, then high-eccentricity migration will remain a viable alternative. On the other hand, if future observations find more systems with aligned obliquities, then it will support the paradigm advanced in this work of a coplanar companion population for cool hot-Jupiter hosts.

\subsection{Inclination of Companions to Hot Jupiters around Hot Stars}

In this paper, we only consider the inclinations of distant companions to hot Jupiters around cool stars because their obliquity angles are conveniently well aligned, making this type of analysis possible. This raises the question, "Are companions to hot Jupiters around hot stars also coplanar?"

One possibility is that distant companions to hot Jupiters around hot stars are not well aligned with the hot Jupiters' orbits, and that their gravitational perturbations either cause or contribute to the the increased scatter in spin/orbit angles that are observed for these stars. This scenario hints at the explanation for correlation between stellar obliquity and stellar effective temperature by Batygin (2012), who suggests that the increased prevalence of stellar companions for more massive stars can explain the misalignment of hot-Jupiter orbits with the spin axes of hot stars. Batygin (2012) suggests that torques from distant, misaligned companions on the protoplanetary disks can cause the misalignments that are observed; our results demonstrate the well-known (e.g., Lai \& Pu 2017) result that closer misaligned companions can cause misalignments via secular interactions with the planet itself.

Another possibility is that most distant companions to hot Jupiters around stars of all temperatures and masses are roughly coplanar with the hot Jupiters, and the large scatter in stellar obliquity observed in hot stars comes from some other mechanism. In this case, although the companions do not disturb the hot Jupiters' spin/orbit angles, we cannot tell because there is no apparent pattern for distant companions to disrupt.

\subsection{Caveats}

The major caveats on the results quoted above can be summarized as follows. First, even in this paradigm, individual systems containing hot Jupiters around cool stars could (rarely) be found to have high-inclination companions due to unusual dynamical histories. For this reason, the methods and results of this work provide a statistical statement on the population of companions to hot Jupiters around cool stars, and cannot be used to determine true inclinations for individual systems.

Second, the temperature cut-off that we use in this work is chosen based on effective temperature. As these measurements are improved, some systems may move into or out of our sample. The ideal way to define the sample would be to include stars with thick convective envelopes, but currently, effective temperature is the best proxy for envelope size. As such, systems with host stars close to the temperature cut-off may be incorrectly categorized.

Third, only dynamically coupled companions can be included in analyses of this nature. Companions with sufficiently large orbital radii may become decoupled from the dynamics of the inner system, and no longer affect the orbital precession of the hot Jupiter. Our statistical result does not apply to these very distant decoupled companions. Field surveys indicate that the occurrence rate of brown dwarfs (with masses ranging from 13-80 $M_{J}$ ) around Sun-like stars is low (Ma \& Ge 2014), with exact fractions ranging from $0.6 \%$ to $0.8 \%$ (Vogt et al. 2002; Patel et al. 2007; Wittenmyer et al. 2009; Sahlmann et al. 2011), suggesting that the companions for which we do not have fitted orbits (HAT-P-4, WASP-22, 
WASP-52) are more likely to be planetary companions rather than distant (potentially decoupled) brown dwarfs.

\section{Conclusion}

In this work, we have shown statistically that distant exterior companions to hot Jupiters around cool stars must typically orbit in roughly the same plane as the hot Jupiter itself. Specifically, companion orbits must generally fall within $20^{\circ}-30^{\circ}$ of the plane containing the hot Jupiter (see Figure 4). ${ }^{9} \mathrm{We}$ constructed a sample of six hot-Jupiter systems around cool stars (specifically, HAT-P-4, HAT-P-13, WASP-22, WASP-41, WASP-47, and WASP-53) and calculated the dynamical effects of distant perturbing companions as a function of the companion's orbital inclination. We performed a large ensemble of numerical simulations to show that if the inclination distribution companions to these systems extended much more than $20^{\circ}$ away from coplanar, then we would have been unlikely to observe the measured obliquities in our sample. We have also used secular theory for comparison; this approach is in good agreement with the full $\mathrm{N}$-body simulations and can provide a time-saving alternative (see Figure 3).

The fact that companions to hot Jupiters tend to orbit in nearly the same plane as the hot Jupiters themselves disfavors formation and migration models involving planet/planet scattering for hot Jupiters around cool stars. In particular, Kozai-Lidov migration typically requires a perturbing planetary (or stellar) companion with a mutual inclination of about $40^{\circ}$ or more. Mutual inclinations this large are strongly disfavored by our statistical analysis. This finding —along with the fact that too few highly eccentric proto-hot Jupiters have been detected in Kepler data to explain the hot-Jupiter population (Dawson et al. 2015) —suggests that Kozai-Lidov migration is not the dominant mode for forming hot-Jupiter systems. Instead, this result favors formation scenarios that take place mostly within the plane of the protoplanetary disk, such as disk migration, in situ formation, or in some cases, coplanar high-eccentricity migration.

We thank Gongjie Li, Heather Knutson, Josh Winn, Ben Montet, Danielle Piskorz, Sarah Millholland, Clara Eng, and Iryna Butsky for useful conversations. We also thank Michael Dieterle for visualization suggestions, and thank the referee, Chris Spalding, for prompt and useful feedback. J.C.B. and A.V. are supported by the NSF Graduate Research Fellowship grant Nos. DGE 1256260 and 1144152, respectively. This work was performed in part under contract with the California Institute of Technology (Caltech)/Jet Propulsion Laboratory (JPL) funded by NASA through the Sagan Fellowship Program executed by the NASA Exoplanet Science Institute. This work used both the Extreme Science and Engineering Discovery Environment (XSEDE; NSF grant number ACI-1053575) and resources provided by the Open Science Grid, which is supported by the National Science Foundation and the U.S. Department of Energy's Office of Science.

Note Added in Proof. In our preliminary integrations, we tested the effect of a stellar J2 on the results presented herein. For the systems considered in this work, the effect is negligible, and so we did not include $\mathrm{J} 2$ in the integrations used to make Figure 4 (in order to not

\footnotetext{
9 Note that we expect any additional planets to also be roughly in the same plane (see, for example, WASP-47).
}

add another variable to the problem). After the acceptance of this paper, we have performed additional integrations to test this assumption, and confirmed that including $\mathrm{J} 2$ does not change the results of this paper. As stated in Section 3.4, the range of applicability of our conclusions has some limits, which will depend on the stellar J2 as well as other dynamical factors (so that other systems may be affected by J2). In future work, we plan to evaluate these limits.

\section{ORCID iDs}

Juliette C. Becker (1) https://orcid.org/0000-0002-7733-4522 Andrew Vanderburg (1) https://orcid.org/0000-00017246-5438

Fred C. Adams ํㅏ https://orcid.org/0000-0002-8167-1767

Tali Khain (1) https://orcid.org/0000-0001-7721-6457

Marta Bryan (i) https://orcid.org/0000-0002-6076-5967

\section{References}

Adams, F. C., \& Bloch, A. M. 2015, MNRAS, 446, 3676

Albrecht, S., Winn, J. N., Johnson, J. A., et al. 2012, ApJ, 757, 18

Anderson, D. R., Collier Cameron, A., Gillon, M., et al. 2011, A\&A, 534, A16

Bakos, G. Á., Howard, A. W., Noyes, R. W., et al. 2009, ApJ, 707, 446

Batygin, K. 2012, Natur, 491, 418

Batygin, K., Bodenheimer, P. H., \& Laughlin, G. P. 2016, ApJ, 829, 114

Becker, J. C., \& Adams, F. C. 2017, MNRAS, 468, 549

Becker, J. C., \& Batygin, K. 2013, ApJ, 778, 100

Becker, J. C., Vanderburg, A., Adams, F. C., Rappaport, S. A., \& Schwengeler, H. M. 2015, ApJL, 812, L18

Bryan, M. L., Knutson, H. A., Howard, A. W., et al. 2016, ApJ, 821, 89

Burke, C. J., McCullough, P. R., Valenti, J. A., et al. 2007, ApJ, 671, 2115 Chambers, J. E. 1999, MNRAS, 304, 793

Cumming, A., Butler, R. P., Marcy, G. W., et al. 2008, PASP, 120, 531

Damasso, M., Biazzo, K., Bonomo, A. S., et al. 2015, A\&A, 575, A111

Dawson, R. I., Murray-Clay, R. A., \& Johnson, J. A. 2015, ApJ, 798, 66

Fabrycky, D., \& Tremaine, S. 2007, ApJ, 669, 1298

Fabrycky, D. C., \& Winn, J. N. 2009, ApJ, 696, 1230

Fielding, D. B., McKee, C. F., Socrates, A., Cunningham, A. J., \& Klein, R. I. 2015, MNRAS, 450, 3306

Fressin, F., Torres, G., Charbonneau, D., et al. 2013, ApJ, 766, 81

Hellier, C., Anderson, D. R., Collier Cameron, A., et al. 2012, MNRAS, 426, 739

Huang, C., Wu, Y., \& Triaud, A. H. M. J. 2016, ApJ, 825, 98

Hut, P. 1980, A\&A, 92, 167

Johnson, M. C., Cochran, W. D., Addison, B. C., Tinney, C. G., \& Wright, D. J. 2017, AJ, in press, arXiv:1708.01291

Kley, W., \& Nelson, R. P. 2012, ARA\&A, 50, 211

Knutson, H. A., Fulton, B. J., Montet, B. T., et al. 2014, ApJ, 785, 126

Kovács, G., Bakos, G. Á., Torres, G., et al. 2007, ApJL, 670, L41

Kozai, Y. 1962, AJ, 67, 591

Kraft, R. P. 1967, ApJ, 150, 551

Lai, D. 2012, MNRAS, 423, 486

Lai, D. 2014, MNRAS, 440, 3532

Lai, D., Foucart, F., \& Lin, D. N. C. 2011, MNRAS, 412, 2790

Lai, D., \& Pu, B. 2017, AJ, 153, 42

Lee, E. J., Chiang, E., \& Ormel, C. W. 2014, ApJ, 797, 95

Lidov, M. L. 1962, P\&SS, 9, 719

Ma, B., \& Ge, J. 2014, MNRAS, 439, 2781

Marcy, G., Butler, R. P., Fischer, D., et al. 2005, PThPS, 158, 24

Maxted, P. F. L., Anderson, D. R., Collier Cameron, A., et al. 2011, PASP, 123,547

Maxted, P. F. L., Anderson, D. R., Gillon, M., et al. 2010, AJ, 140, 2007

Mayor, M., Marmier, M., Lovis, C., et al. 2011, A\&A, submitted, arXiv: 1109.2497

Mazeh, T., Perets, H. B., McQuillan, A., \& Goldstein, E. S. 2015, ApJ, 801, 3

Morton, T. D., \& Winn, J. N. 2014, ApJ, 796, 47

Murray, C. D., \& Dermott, S. F. 1999, Solar System Dynamics (Cambridge: Cambridge Univ. Press)

Nagasawa, M., Ida, S., \& Bessho, T. 2008, ApJ, 678, 498

Naoz, S., Farr, W. M., Lithwick, Y., Rasio, F. A., \& Teyssandier, J. 2011, Natur, 473, 187

Neveu-VanMalle, M., Queloz, D., Anderson, D. R., et al. 2016, A\&A, 586, A93

Ngo, H., Knutson, H. A., Hinkley, S., et al. 2015, ApJ, 800, 138 
Ohta, Y., Taruya, A., \& Suto, Y. 2005, ApJ, 622, 1118

Patel, S. G., Vogt, S. S., Marcy, G. W., et al. 2007, ApJ, 665, 744

Petrovich, C. 2015, ApJ, 805, 75

Piskorz, D., Knutson, H. A., Ngo, H., et al. 2015, ApJ, 814, 148

Sahlmann, J., Ségransan, D., Queloz, D., et al. 2011, A\&A, 525, A95

Sanchis-Ojeda, R., Rappaport, S., Pallé, E., et al. 2015a, ApJ, in press, arXiv: 1504.04379

Sanchis-Ojeda, R., Winn, J. N., Dai, F., et al. 2015b, ApJL, 812, L11

Southworth, J., Tregloan-Reed, J., Andersen, M. I., et al. 2016, MNRAS, 457,4205

Spalding, C., \& Batygin, K. 2015, ApJ, 811, 82

Spalding, C., \& Batygin, K. 2017, AJ, 154, 93

Spalding, C., Batygin, K., \& Adams, F. C. 2014, ApJL, 797, L29

Steffen, J. H., Ragozzine, D., Fabrycky, D. C., et al. 2012, PNAS, 109, 7982

Stevenson, D. J. 1982, P\&SS, 30, 755

Tanaka, H., Takeuchi, T., \& Ward, W. R. 2002, ApJ, 565, 1257
Tremaine, S., \& Dong, S. 2012, AJ, 143, 94

Triaud, A. H. M. J., Neveu-VanMalle, M., Lendl, M., et al. 2017, MNRAS, 467, 1714

van Saders, J. L., \& Pinsonneault, M. H. 2013, ApJ, 776, 67

Vanderburg, A., Becker, J. C., Buchhave, L. A., et al. 2017, AJ, in press (arXiv:1710.00026)

Vogt, S. S., Butler, R. P., Marcy, G. W., et al. 2002, ApJ, 568, 352

Wang, J., Fischer, D. A., Horch, E. P., \& Huang, X. 2015, ApJ, 799, 229

Weiss, L. M., Deck, K. M., Sinukoff, E., et al. 2017, AJ, 153, 265

Winn, J. N., Fabrycky, D., Albrecht, S., \& Johnson, J. A. 2010a, ApJL, 718, L145

Winn, J. N., Howard, A. W., Johnson, J. A., et al. 2011, AJ, 141, 63

Winn, J. N., Johnson, J. A., Howard, A. W., et al. 2010b, ApJ, 718, 575

Wittenmyer, R. A., Endl, M., Cochran, W. D., et al. 2009, AJ, 137, 3529

Wright, J. T., Marcy, G. W., Howard, A. W., et al. 2012, ApJ, 753, 160

Zhou, G., Bakos, G. Á, Hartman, J. D., et al. 2017, AJ, 153, 211 